\title{
Data integration and evolutionary analysis of long non-coding RNAs in 25 flowering plants
}

Shiye Sang ${ }^{1,2}$, Wen Chen ${ }^{1}$, Di Zhang ${ }^{1,2}$, Xuan Zhang ${ }^{1,2}$, Wenjing Yang ${ }^{1,2}$ and Changning Liu ${ }^{1,3,4^{*}}$

From 19th International Conference on Bioinformatics 2020 (InCoB2020)

Virtual. 25-29 November 2020

\begin{abstract}
Background: Long non-coding RNAs (IncRNAs) play vital roles in many important biological processes in plants. Currently, a large fraction of plant IncRNA studies center at IncRNA identification and functional analysis. Only a few plant IncRNA studies focus on understanding their evolutionary history, which is crucial for an in-depth understanding of IncRNAs. Therefore, the integration of large volumes of plant IncRNA data is required to deeply investigate the evolution of IncRNAs.

Results: We present a large-scale evolutionary analysis of IncRNAs in 25 flowering plants. In total, we identified 199,796 high-confidence IncRNAs through data integration analysis, and grouped them into 5497 IncRNA orthologous families. Then, we divided the IncRNAs into groups based on the degree of sequence conservation, and quantified the various characteristics of 756 conserved Arabidopsis thaliana IncRNAs. We found that compared with non-conserved IncRNAs, conserved IncRNAs might have more exons, longer sequence length, higher expression levels, and lower tissue specificities. Functional annotation based on the A. thaliana coding-IncRNA gene co-expression network suggested potential functions of conserved IncRNAs including autophagy, locomotion, and cell cycle. Enrichment analysis revealed that the functions of conserved IncRNAs were closely related to the growth and development of the tissues in which they were specifically expressed.
\end{abstract}

\footnotetext{
* Correspondence: liuchangning@xtbg.ac.cn

${ }^{1}$ CAS Key Laboratory of Tropical Plant Resources and Sustainable Use,

Xishuangbanna Tropical Botanical Garden, Chinese Academy of Sciences, Menglun, Mengla 666303, Yunnan, China

${ }^{3}$ Center of Economic Botany, Core Botanical Gardens, Chinese Academy of Sciences, Menglun, Mengla 666303, Yunnan, China

Full list of author information is available at the end of the article
}

(c) The Author(s). 2021 Open Access This article is licensed under a Creative Commons Attribution 4.0 International License, which permits use, sharing, adaptation, distribution and reproduction in any medium or format, as long as you give appropriate credit to the original author(s) and the source, provide a link to the Creative Commons licence, and indicate if changes were made. The images or other third party material in this article are included in the article's Creative Commons licence, unless indicated otherwise in a credit line to the material. If material is not included in the article's Creative Commons licence and your intended use is not permitted by statutory regulation or exceeds the permitted use, you will need to obtain permission directly from the copyright holder. To view a copy of this licence, visit http://creativecommons.org/licenses/by/4.0/. The Creative Commons Public Domain Dedication waiver (http://creativecommons.org/publicdomain/zero/1.0/) applies to the data made available in this article, unless otherwise stated in a credit line to the data. 
Conclusions: Comprehensive integration of large-scale IncRNA data and construction of a phylogenetic tree with orthologous IncRNA families from 25 flowering plants was used to provide an oversight of the evolutionary history of plant IncRNAs including origin, conservation, and orthologous relationships. Further analysis revealed a differential characteristic profile for conserved IncRNAs in A. thaliana when compared with non-conserved IncRNAs. We also examined tissue specific expression and the potential functional roles of conserved IncRNAs. The results presented here will further our understanding of plant IncRNA evolution, and provide the basis for further in-depth studies of their functions.

Keywords: IncRNAs, Flowering plants, Evolutionary analysis, Conservation, Arabidopsis thaliana, Co-expression network

\section{Background}

Long non-coding RNAs (lncRNAs) are a class of noncoding RNAs longer than $200 \mathrm{nt}$ with high tissue specificities. They can be divided into intergenic, intronic, sense and antisense lncRNA transcripts based on their relative position to coding genes [1]. In recent years, a variety of methods based on machine learning algorithms have greatly improved the performance of IncRNA identification and functional annotation, and made lncRNA function study in diverse species receive extensive attention, such as the significant roles of lncRNAs in tumorigenesis and cancer progression, and in plant development and stress responses [2-5]. The sequence homology and conservation of lncRNAs provide insights into their functions. Therefore, there is a need to understand the evolutionary dynamics of lncRNAs.

In animals, great breakthroughs have been made in IncRNA evolutionary analysis, which is of great benefit to the understanding of the functions of animal lncRNAs and the evolution of regulatory networks in which they are involved. Washietl et al. found that mammalian long intergenic non-coding RNAs (lincRNAs) show strong conservation of tissue specificities and higher primary sequence conservation in promoters and exons than in evolutionarily young lincRNAs [6]. An analysis of evolutionary age and lncRNA families in tetrapods showed that ancient lncRNAs, which were generally actively regulated, might play a major role in embryonic development, and conserved IncRNAs probably function in fundamental processes including spermatogenesis and synaptic transmission [7]. Interestingly, Hezroni et al. reported that the conserved functions of lincRNAs required only short patches of specific sequences and could withstand major changes in gene structure [8].

In plants, no large-scale comprehensive evolutionary analyses of lncRNAs have been performed in multiple species, but some attempts have been made. A comparison between $A$. thaliana lincRNAs and genomic sequences of the other six plant species showed that only $2 \%$ of lincRNAs displayed evolutionary conservation [9]. Additionally, $\mathrm{Li}$ et al. revealed that only $25 \%$ of the IncRNAs identified in maize (Zea mays) could find homologous in sorghum (Sorghum bicolor) [10]. A recent study reported that 575 orthologous lncRNA pairs were identified between A. thaliana and Arabidopsis lyrata, while few orthologous lncRNA pairs were identified in rice (Oryza sativa) and it's four related species [11].

A large number of lncRNAs have been discovered in various plants. LncRNA data resources are rapidly growing, and have made it possible to use bioinformatics methods and tools to collect and integrate lncRNA data to study its evolution and function. Here, we conducted a large-scale evolutionary analysis of lncRNAs from 25 flowering plants. Through data integration analysis of four public lncRNA databases, we identified 199,796 high confidence lncRNAs, and classified them into orthologous families. We grouped these lncRNAs based on their sequence conservation, and compared the sequence, structure and expression differences between conserved and non-conserved IncRNAs to produce a comprehensive profile of conserved plant lncRNAs. This work allows us to better understand the evolution of plant lncRNAs, and provides valuable clues for further in-depth studies of plant lncRNA functions.

\section{Results}

\section{Comprehensive collection and integration of IncRNAs in 25 flowering plants}

To analyze plant lncRNAs as comprehensively as possible, we used an analysis pipeline with three main parts: 1) lncRNA data collection and integration; 2) lncRNA sequence conservation analysis; and 3) lncRNA functional annotation. For the first part, we collected lncRNAs from four comprehensive and reliable public plant lncRNA databases, including CANTATAdb2.0, GreeNC, RefSeq, and NONCODE. LncRNA sequences were then filtered based on sequence length (longer than $200 \mathrm{nt}$ ), coding potential (calculated by CPC2) and genome location (identified by Gffcompare). Based on the amount of data, and the quality of the genomes and genome annotations, we further selected 25 species for subsequent analysis. The obvious overlap between different 
data sources required us to integrate the data from different databases to obtain a non-redundant lncRNA dataset. This was the key step in the first part of our pipeline. The lncRNA sequences were mapped to specified genome versions of the plants to determine their exact genomic locations, and redundant lncRNAs were removed if their locations were highly overlapping (Additional file 1). As a result, we obtained a final high confidence lncRNA dataset containing 199,796 lncRNA transcripts (Table 1).

In the 25 species examined, lncRNAs of seven species came from two databases, lncRNAs of 17 species came from three databases, and lncRNAs of $A$. thaliana came from four databases. Moreover, there was a 6.85 -fold variation in the numbers of IncRNAs in different plants, from 3430 in Citrus sinensis to 23,512 in Zea mays. Species with more than 10,000 lncRNAs also include Brassica napus, Brassica rapa, and Medicago truncatula, while other species each have lncRNAs in the thousands.

\section{Plant IncRNA sequence conservation}

Putative lncRNA orthologous families were identified in plants through multi-species comparison. BLASTN was used to perform pairwise alignment of lncRNA sequences between species [12]. IncRNA orthologous pairs were identified through reciprocal best hits, and they were connected using the single-linkage clustering method to construct IncRNA families. To further explore the evolutionary conservation of plant lncRNA, all lncRNA families were located on a phylogenetic tree made by TimeTree according to which species does lncRNAs in them belong to (Fig. 1) [13].

The phylogenetic tree revealed that the evolution of the IncRNAs in these 25 species spans 180 Myr. We identified 5497 lncRNA families with a total of 13,564 conserved lncRNAs. Among these lncRNA families, 1953 (35.5\%) families had a total of 6269 (46.22\%) conserved lncRNAs that originated more than $50 \mathrm{Myr}$ ago, 1401 (25.49\%) families had a total of 5171 (38.12\%) conserved lncRNAs that originated more than $100 \mathrm{Myr}$ ago,

Table 1 IncRNA data integration results

\begin{tabular}{|c|c|c|c|c|c|c|}
\hline Species & GREENC & CANTATADB & RefSeq & NONCODE & Number of IncRNAs collected & Number of unique IncRNAs \\
\hline Amborella trichopoda & 5698 & 5511 & 4750 & 0 & 15,959 & 7074 \\
\hline Arabidopsis lyrata & 4363 & 7593 & 5311 & 0 & 17,267 & 9363 \\
\hline Arabidopsis thaliana & 3008 & 4373 & 4083 & 3763 & 15,227 & 5539 \\
\hline Brachypodium distachyon & 5584 & 4945 & 8779 & 0 & 19,308 & 6783 \\
\hline Brassica napus & 0 & 12,010 & 18,114 & 0 & 30,124 & 16,597 \\
\hline Brassica rapa & 0 & 8501 & 10,102 & 0 & 18,603 & 10,797 \\
\hline Citrus sinensis & 2562 & 0 & 6581 & 0 & 9143 & 3430 \\
\hline Cucumis sativus & 1929 & 7348 & 1639 & 0 & 10,916 & 5466 \\
\hline Fragaria vesca & 3503 & 0 & 5007 & 0 & 8510 & 3889 \\
\hline Glycine max & 6689 & 3096 & 10,980 & 0 & 20,765 & 8817 \\
\hline Gossypium raimondii & 4216 & 0 & 12,765 & 0 & 16,981 & 6422 \\
\hline Malus domestica & 4126 & 10,924 & 11,608 & 0 & 26,658 & 9228 \\
\hline Manihot esculenta & 3468 & 9504 & 4874 & 0 & 17,846 & 7660 \\
\hline Medicago truncatula & 9676 & 3590 & 3874 & 0 & 17,140 & 10,904 \\
\hline Musa acuminata & 4071 & 3001 & 5428 & 0 & 12,500 & 5121 \\
\hline Oryza brachyantha & 0 & 6004 & 2720 & 0 & 8724 & 4926 \\
\hline Oryza sativa & 5237 & 2788 & 10,090 & 0 & 18,115 & 7211 \\
\hline Populus trichocarpa & 5569 & 4322 & 0 & 0 & 9891 & 8334 \\
\hline Prunus persica & 3301 & 2902 & 7274 & 0 & 13,477 & 4183 \\
\hline Solanum lycopersicum & 3440 & 4716 & 2203 & 0 & 10,359 & 6807 \\
\hline Solanum tuberosum & 6680 & 5790 & 7727 & 0 & 20,197 & 7797 \\
\hline Sorghum bicolor & 5305 & 2600 & 4821 & 0 & 12,726 & 6326 \\
\hline Theobroma cacao & 4268 & 5256 & 9026 & 0 & 18,550 & 7459 \\
\hline Vitis vinifera & 2526 & 4542 & 5340 & 0 & 12,408 & 6151 \\
\hline Zea mays & 18,110 & 10,761 & 11,543 & 0 & 40,414 & 23,512 \\
\hline
\end{tabular}




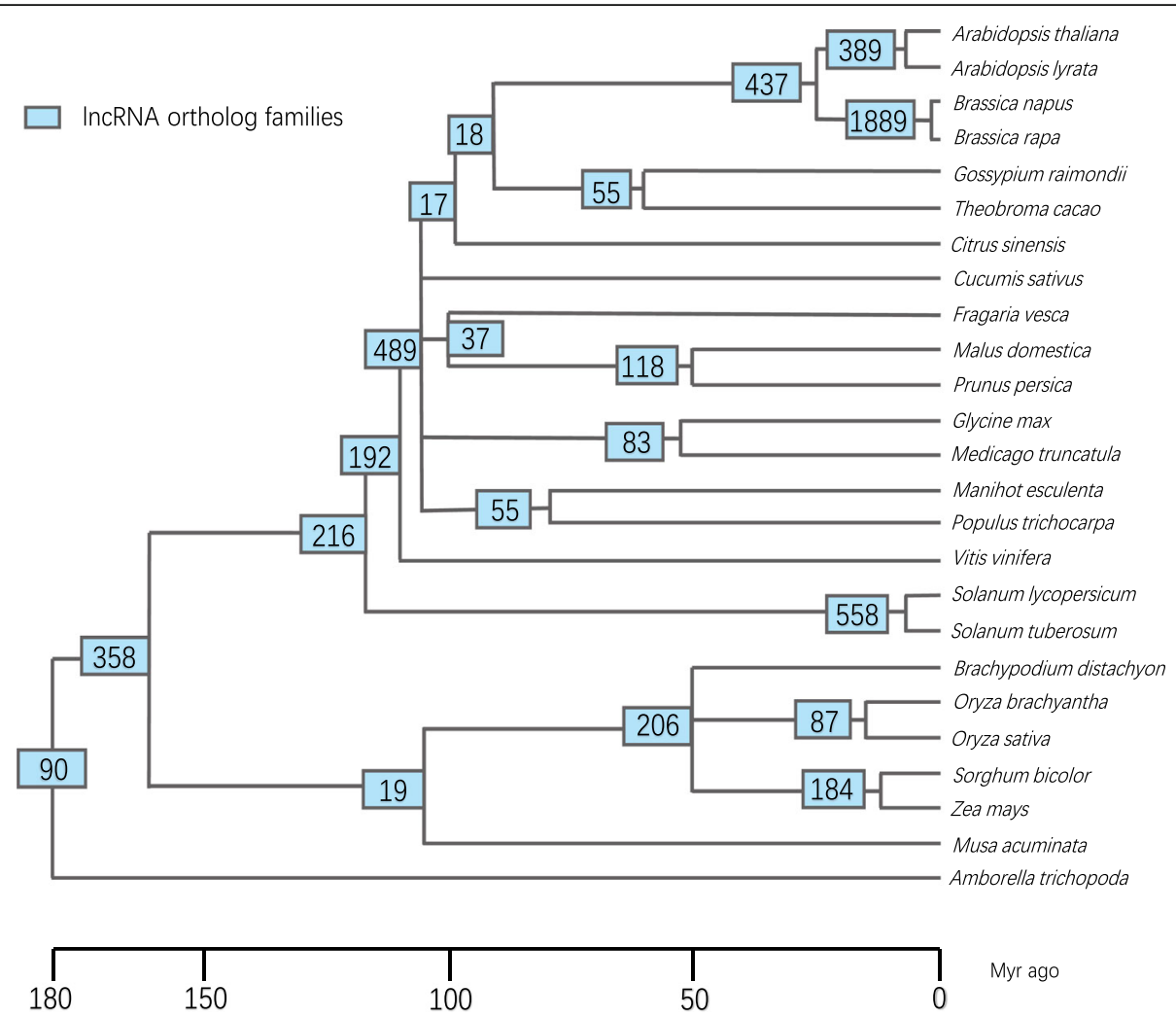

Fig. 1 The phylogenetic tree of IncRNA orthologous families. The numbers in the blue box at the internal branches and roots represent the number of IncRNA orthologous families

448 (8.1\%) families had a total of 2381 (17.55\%) conserved lncRNAs that originated more than $150 \mathrm{Myr}$ ago, and 90 (1.64\%) families had a total of $692(5.10 \%)$ conserved lncRNAs that originated more than 180 Myr ago.

More detailed statistics revealed that only $6.79 \%$ $(13,564$ lncRNAs) of the collected lncRNAs $(199,796$ lncRNAs) were conserved across the plant kingdom. In A. thaliana, the percentage of conserved lncRNAs was $15.56 \%$. The highest proportion of conserved lncRNAs was observed in Brassica rapa (21.01\%) and the lowest in Amborella trichopoda (1.30\%) (Additional file 2). These findings suggest that plant lncRNAs have a fast evolutionary rate, resulting in poor sequence conservation. Further investigation of the proportion of conserved lncRNAs at each branch point revealed that the proportion of conserved lncRNAs among related species (within the same genus) tends to be larger than the proportion of conserved lncRNAs between distant species (between the genera or more distant relatives). The number of orthologous families between Brassica napus and Brassica rapa is 1889, and their percentage of conserved lncRNAs is the highest (13.79\%). This indicates that most IncRNAs were traced to more closely related ancestors, and makes us guess that
lncRNAs between closely related species were more likely to be conserved.

\section{Differences between Arabidopsis IncRNAs with conserved and non-conserved sequences}

A. thaliana is a representative model plant with a highquality genome and in which the lncRNAs identified have the advantages of reliability and accuracy. Therefore, understanding the evolutionary history of A. thaliana lncRNAs is a key step to further our understanding of the plant lncRNA evolution. We divided $A$. thaliana lncRNAs into five categories based on the degree of conservation: "AD-conserved" IncRNAs conserved in Arabidopsis; "BC-conserved" IncRNAs conserved in Brassicaceae; "DL-conserved" lncRNAs conserved in dicotyledons; "AP-conserved" IncRNAs conserved in angiosperms; "non-conserved" lncRNAs with no conservation. DL-conserved and AP-conserved lncRNAs were combined to avoid statistical errors caused by their small sizes. Collectively these groups are referred to as "Ultra-conserved" lncRNAs. Next, we performed a characteristic analysis across the four categories: Nonconserved, AD-conserved, BC-conserved, and Ultraconserved. lncRNA number, length, exon number, 

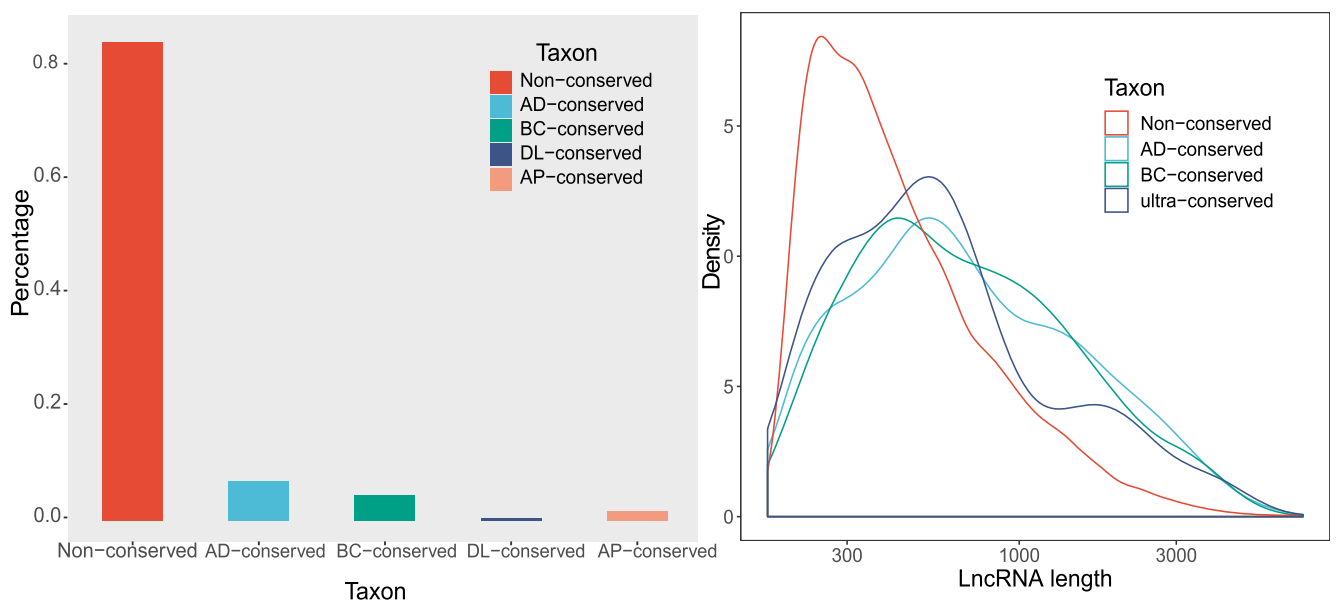

b

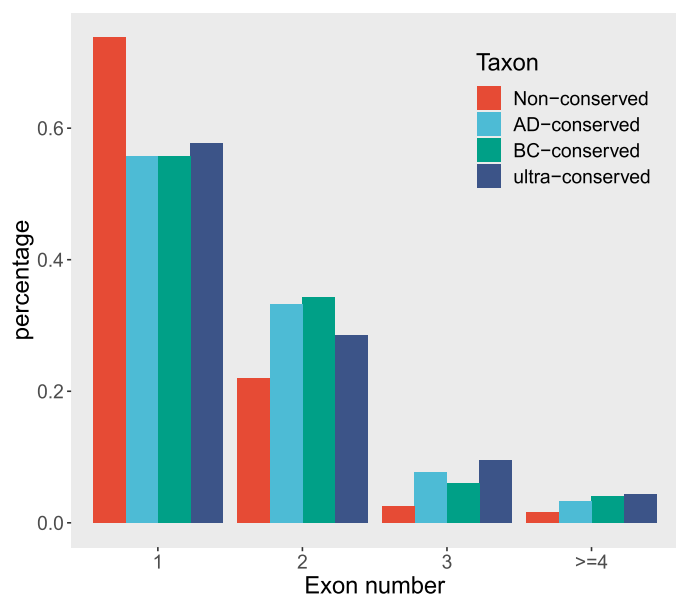

C

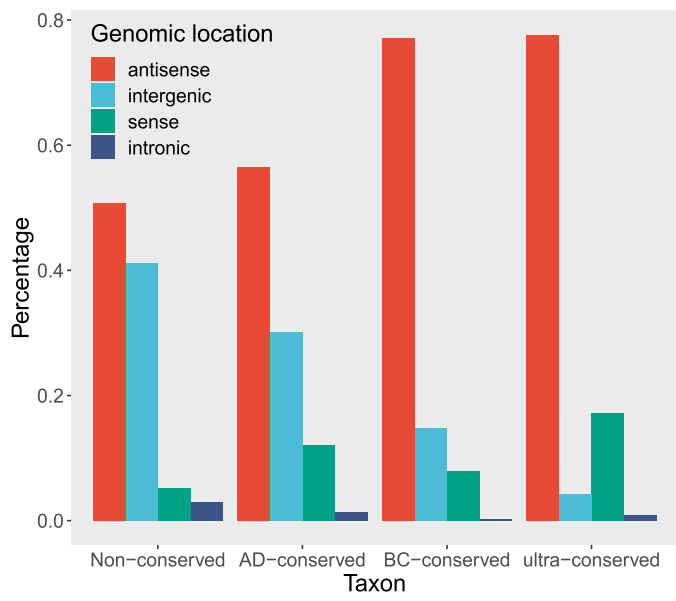

e

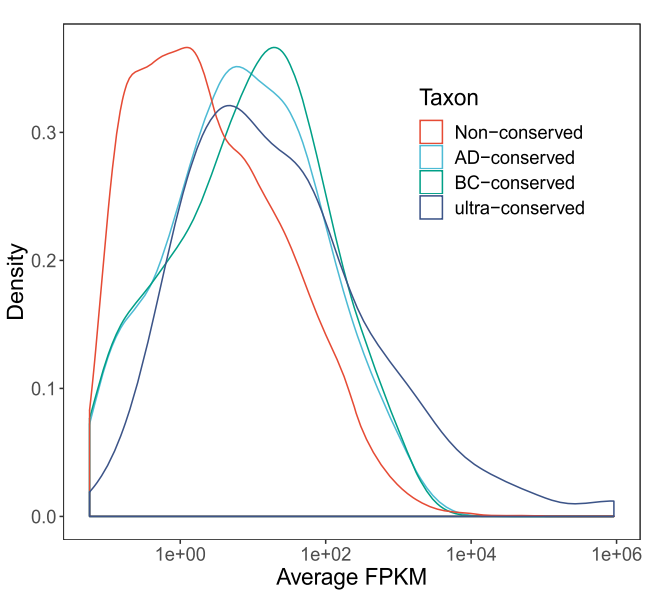

d

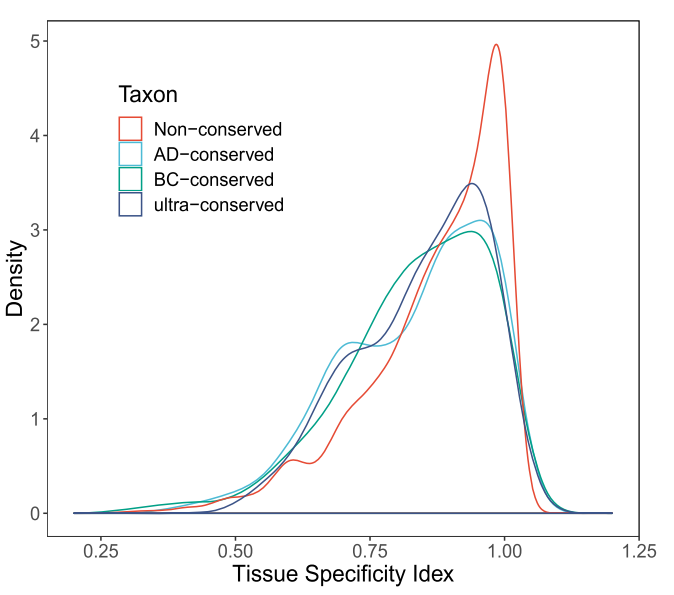

f

Fig. 2 IncRNA classification characteristics. a The number of IncRNAs. b IncRNA length. c The number of IncRNA exons. d Average expression levels of IncRNA. e IncRNA genomic location. $\mathbf{f}$ IncRNA tissue specificity index 
genomic location, average expression levels (AEL), and tissue specificity index ( $\tau$ value) were counted separately (Fig. 2).

There were 4677 non-conserved lncRNAs, which accounted for $84.4 \%$ of the total lncRNAs (Fig. 2a). There were $389 \mathrm{AD}$-conserved and $251 \mathrm{BC}$-conserved lncRNAs, accounting for 7 and $4.5 \%$ of the total, respectively. The 28 DL-conserved and 88 AP-conserved IncRNAs were relatively low and accounted for only $2.1 \%$ of the total.

Examination of lncRNA sequence length and intron/ exon structure revealed that conserved lncRNAs were longer length and contained more exons than did nonconserved lncRNAs. Statistics on the lengths of the lncRNAs across the four categories showed that the density curve of conserved lncRNAs was shifted to the right of that of non-conserved lncRNAs. This result is consistent with the descriptive statistics results that showed the average length of conserved lncRNAs (898 bp) was much longer than that of non-conserved lncRNAs (550 bp) (Fig. 2b). Additionally, all three conserved lncRNA curves showed heavy-tailed distribution. Analysis of the exon numbers across the four lncRNA categories showed that the non-conserved group had a higher proportion of single exon lncRNAs than did the other three groups (Fig. 2c). Conversely, the proportion of multiple exon lncRNAs in conserved lncRNA groups was higher than that in the non-conserved group, and Ultra-conserved lncRNAs accounted for the highest proportion of lncRNAs with exon numbers equal to or greater than three.

lncRNA expression was examined by firstly calculating average expression levels (AEL), where high values reflect high lncRNAs expression levels. The ratio of highly expressed lncRNAs in conserved lncRNAs was much higher than that in non-conserved lncRNAs, and the ratio of highly expressed lncRNAs in the three categories of conserved lncRNAs increased slightly with increased conservation degrees (Fig. 2d). We also calculated the tissue specificity index ( $\tau$ value), where high values reflect high lncRNAs tissue specificities. We observed a higher proportion of tissue-specific expression in nonconserved lncRNAs than in conserved lncRNAs (Fig. 2f). Using a $\tau$ value 0.9 as the threshold, $51 \%$ of the nonconserved and $39 \%$ of conserved lncRNAs exhibit tissuespecific expression, respectively.

We examined the genomic location of lncRNAs (Fig. 2e) and counted the number of antisense-, intergenic-, sense-, and intronic transcripts in the four categories. The distribution of different transcript types in each lncRNA category were roughly the same, with antisense transcripts accounting for 50.69 to $77.59 \%$ of the total, followed by intergenic transcripts (4.31-41.16\%), sense transcripts $(0.86-2.97 \%)$, and intronic transcripts
$(0-3 \%)$. As the degree of conservation increased, the number of antisense transcripts increased and the number of intergenic transcripts decreased. Therefore, we speculated that conserved lncRNAs might be more likely to function as antisense transcripts.

\section{Functional annotation of conserved IncRNAs based on coding-IncRNA gene co-expression network}

The extent of lncRNAs conservation is generally considered to be the key to evaluating their functions. To shed further light on the biological function of conserved lncRNAs, we annotated the functions of 756 conserved lncRNAs from the AD-conserved, BC-conserved, and Ultra-conserved groups based on the theory that the linked gene-pairs in the coding-lncRNA gene coexpression network tended to have more similar annotated functions [14].

To construct the coding-lncRNA $A$. thaliana gene coexpression network, we used the collected RNA-seq datasets to quantify the lncRNA- and coding-gene expression levels. Spearman's correlation coefficient was used to identify co-expressed gene-pairs from the genes with high expression variation (top $75 \%$ percentile). The $P$-value was calculated by Fisher transformation, and $P$ value sets for each gene were corrected using the Bonferroni method. Only gene-pairs with a corrected $P$ value of 0.05 or less were used for subsequent analyses. Finally, we constructed a coding-lncRNA gene coexpression network with 28,730 coding-genes, 384 lncRNA-genes and 38,146,872 edges. Nearly 287,222 edges $(0.75 \%)$ were linked between coding- and lncRNAgenes, 37,858,201 edges (99.24\%) were linked between coding-genes, and another 1449 edges $(0.0038 \%)$ were connected between pairs of lncRNA-genes.

Each conserved lncRNA was annotated based on its immediate neighbor coding-genes that had previously annotated with at least one gene ontology (GO) Biological Process (BP). Using this approach, we identified 196 of 756 conserved lncRNAs annotated with at least one GO BP term (Additional file 3). We then conducted functional enrichment analysis using a hypergeometric distribution test $(P$-value $<0.05)$ to explore which functions are performed more often in conserved lncRNAs. The results showed that 39 GO BP terms were significantly enriched in conserved lncRNAs (Additional file 4). The top ten GO BP terms $(P$-value $=6.03 \mathrm{e}-06)$ were 'cell junction organization,' 'aging,' autophagy-related (including 'process utilizing autophagic mechanism,' and 'autophagy'), movement-related (including 'locomotion,' 'movement of cell or subcellular component,' and 'cell motility'), and cell-cycle-related (including 'chromosome organization,' 'mitotic cell cycle,' 'cell cycle,' and 'cytoskeleton organization') (Fig. 3). 


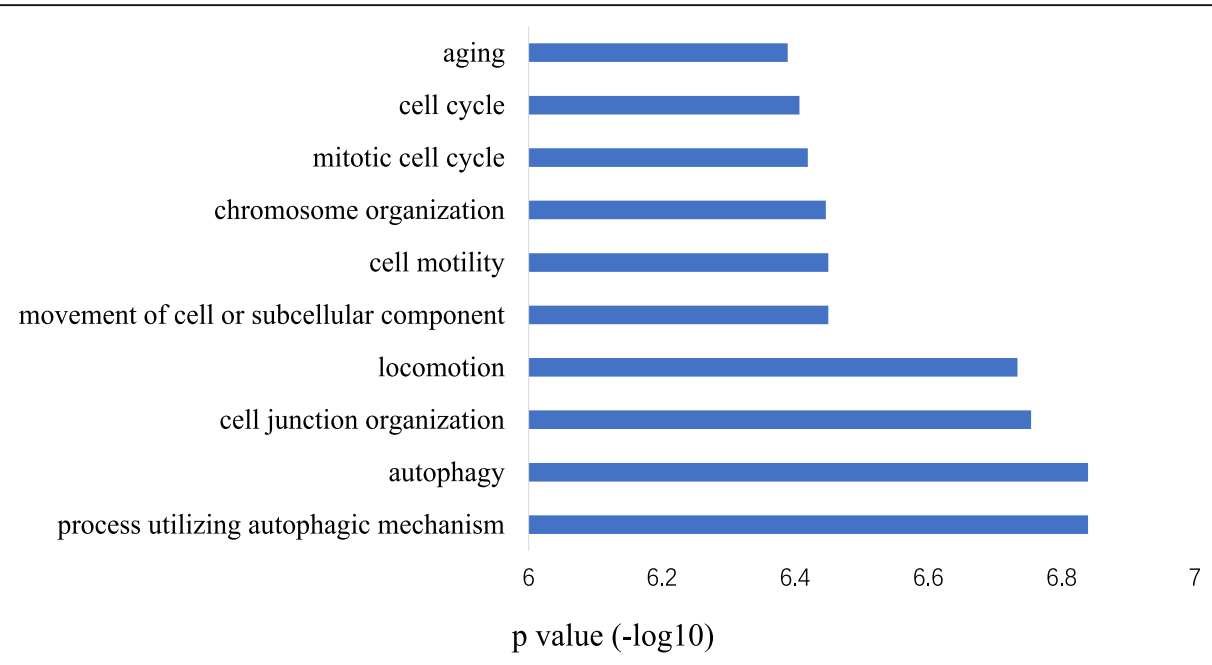

Fig. 3 Gene ontology biological process enrichment results for 196 conserved IncRNAs

Functional enrichment analysis of conserved IncRNAs specifically expressed in certain tissues

Analysis of the expression profiles of the conserved lncRNAs (Fig. 4) revealed that these 756 lncRNAs have obvious tissue specificities. Moreover, their expression patterns fell into nine classes with obvious tissue differences. This phenomenon indicated that the functions of tissue specific lncRNAs were closely related to the growth and development of the corresponding tissues. Therefore, we classified these conserved lncRNAs into nine classes based on the tissue in which they were specifically expressed: leaf-, cotyledons-, floral-bud-,

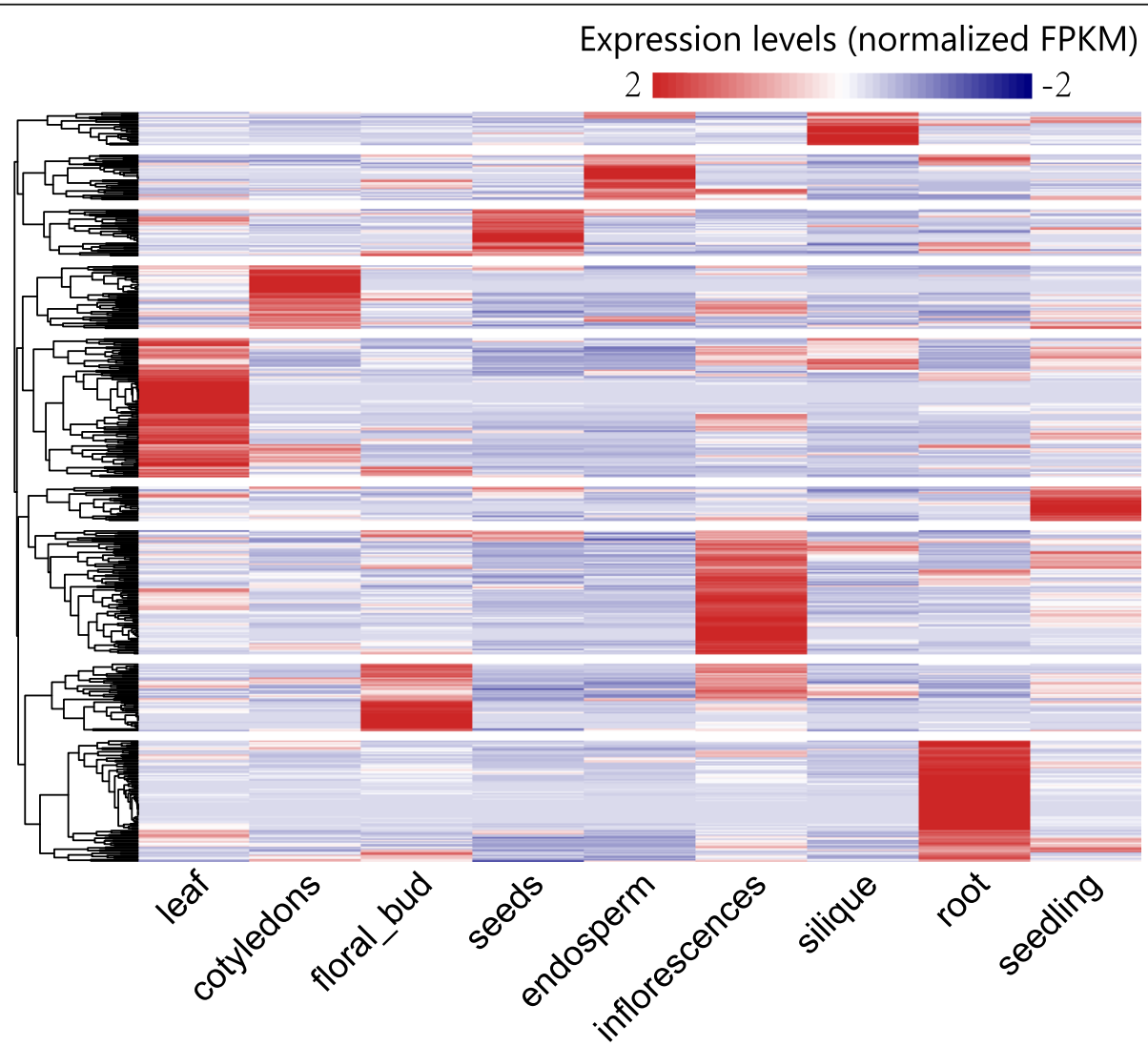

Fig. 4 Heatmap of normalized conserved IncRNAs expression levels in nine tissues 
seedling-, seed-, root-, endosperm-, inflorescence-, and silique-expressed lncRNAs. Functional enrichment analysis was then performed for each lncRNA class.

GO BP enrichment was successful for five of the nine lncRNA classes. We obtained enrichment results of leafexpressed (Fig. 5a), cotyledons-expressed (Fig. 5b), seedexpressed (Fig. 5c), floral-bud-expressed (Fig. 5d), and root-expressed lncRNAs (Fig. 5e). The enriched functions of lncRNAs specifically expressed in different tissues were closely related to the growth and development of those tissues, which are consistent with our previous speculation.

The top ten enriched GO BP terms for leaf-expressed lncRNAs include 'aging,' 'signal transduction,' 'regulation of biological and cellular process,' 'immune system process,' 'cell death,' 'response to stress and stimulus,' 'process utilizing autophagic mechanism,' and 'autophagy.' The enriched GO BP terms for cotyledonsexpressed lncRNAs include terms closely associated with metabolic process, biosynthetic processes, and photosynthesis. Among them, eight BP terms, including 'cellular amino acid metabolic process,' 'organic acid metabolic process,' 'oxoacid metabolic process,' are relevant to metabolism and the other two are 'biosynthetic process' and 'photosynthesis.' The enriched GO BP terms for seed-expressed lncRNAs fit into two categories. The first category is protein-complex-assembly-related BP terms, including 'ribonucleoprotein complex assembly' and 'cellular protein-containing complex assembly.' The second category is metabolic-process-related BP terms, including 'cellular nitrogen compound catabolic process,' 'nucleobase-containing compound catabolic process,' and 'mitochondrion organization.' The top four GO BP terms for floral-bud-expressed lncRNAs are associated with two types of biological processes. One is cellsignaling-related process, including roles like 'cell communication,' 'cell-cell signaling,' and 'signaling.' The other is reproduction processes. For root-expressed IncRNAs, only one GO BP term, 'cell junction organization,' was enriched.

\section{Discussion}

As our understanding of IncRNAs develops, it is clear that the study of a single IncRNA can not inform about the nature of all lncRNAs. In pace with the rapid development of high-throughput sequencing technology, the study of lncRNA evolution dynamics has become one of the most important ways to explore lncRNA function. However, most studies on lncRNA evolution have been performed in animals. Very few studies of lncRNA evolution have been performed in plants, in part because plant lncRNAs have high levels of sequence divergence, there is low genome sequence quality, and the systematic collection and characterization of plant lncRNAs is lacking. Here, through large-scale collection and integration of IncRNA data from multiple databases, we

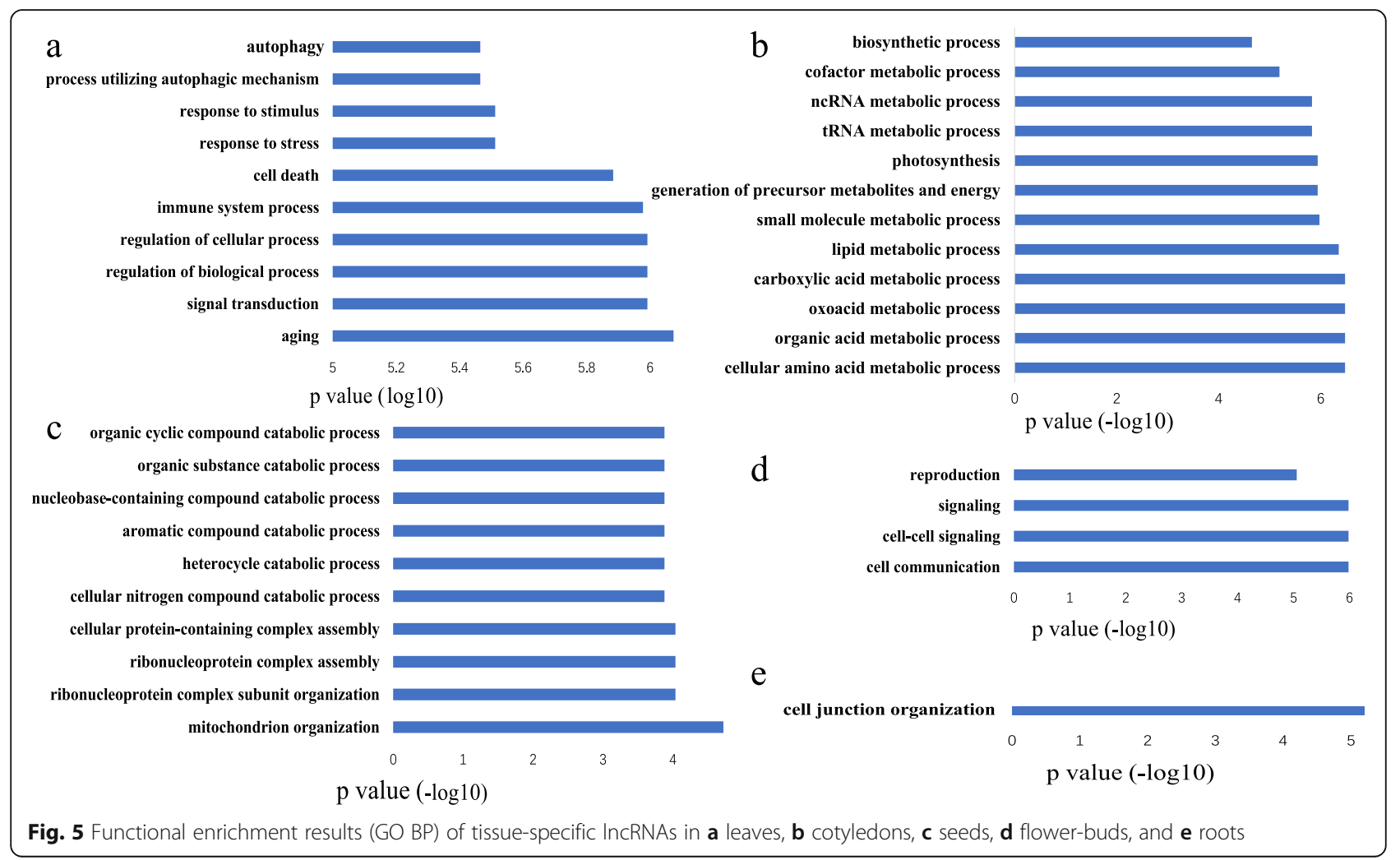


obtained a high-confidence plant lncRNA dataset. Using this dataset, we performed the most comprehensive evolutionary analysis of lncRNAs, including the greatest variety of plant species and the most comprehensive lncRNA sequences to date. Our work can not only lay the foundation for further studying of plant lncRNA evolution but also provide clues for studying the specific function of conserved IncRNAs in different plant species.

Unlike protein-coding genes, IncRNA sequences are poorly conserved. Our results confirm the poor conservation of lncRNAs and are consistent with previous reports of lncRNA conservation in various animals and plants $[8,11,15,16]$. Our results show that the percentage of conserved IncRNA in $A$. thaliana was $15.56 \%$, which is higher than the average proportion of conserved lncRNA in all 25 species of $6.79 \%$. This is probably because we included some species that are closely related to A. thaliana. We found that most lncRNAs were traced back to closer ancestors, and they exhibited more conservation than lncRNAs between distant species. This phenomenon is also reflected in the conservation analyses performed by Deng et al. and Washietl et al. $[6,11]$. While most lncRNAs do not show conservation across the plant kingdom, it is unclear whether lineage-specific lncRNAs play significant roles in lineage-specific biology. Evolutionary analysis of lncRNAs in tetrapods revealed that 425 (3\%) orthologous families originated before $300 \mathrm{Myr}$. Of the plants we studied, only $90(1.64 \%)$ orthologous families originated before 180 Myr. A study on the evolution of lncRNAs in 17 animal species revealed that more than $70 \%$ of IncRNAs appeared before $50 \mathrm{Myr}$, which is significantly different from the results obtained in this study. This discrepancy may suggest that plant lncRNAs are evolutionarily younger than those of animals.

We specially focused on the differences between $A$. thaliana lncRNAs with conserved and non-conserved sequence. Our results showed that conserved lncRNAs have longer sequences, more exons, and higher expression levels than do non-conserved lncRNAs. Since the biological functions of genes are closely related to their structures, we speculate that conserved lncRNAs may be subjected to greater selection pressure during evolution. This selection pressure leads to the evolution of longer sequences and more exons, which are conducive to their roles in regulating plant growth and development, and that these lncRNAs have then evolved to produce higher expression levels. Additionally, our results show that compared with non-conserved lncRNAs, conserved lncRNAs have lower tissue specificity, and are consistent with the results of previous reports $[7,11]$. This finding implies that conserved lncRNAs are more prone to constitutive expression than are non-conserved lncRNAs.
This is potentially due to conserved lncRNAs playing a role in regulatory relationships established early in plant evolution. Additionally, the specific expression of lncRNAs in different tissues reflects that they might play important roles in various stages of plant growth and development.

\section{Conclusions}

Through comprehensive integration of large-scale lncRNA data and construction of a phylogenetic tree using lncRNA orthologous families of 25 flowering plants, we conducted the most thorough investigation of plant lncRNA evolutionary history, which is reflected in the origin, conservation, and orthologous relationships of plant lncRNAs. Our focus on the characteristic differences between conserved and non-conserved lncRNAs provides meaningful insights into the unique traits of conserved and non-conserved lncRNAs including their structure, expression, and genomic location. Further functional analysis of the conserved $A$. thaliana lncRNAs revealed tissue specific expression and potential functional roles. Taken together, these results will better our understanding of the evolutionary mechanisms of lncRNAs in plants and provide a platform for further functional studies.

\section{Methods}

\section{Analysis pipeline}

A three-part analysis pipeline was created: 1) lncRNA data collection and integration; 2) lncRNA sequence conservation analysis; and 3) lncRNA function annotation (Fig. 6). The first part of the pipeline included the collection and integration of plant lncRNAs. The second part of the pipeline included construction of the lncRNA ortholog families of 25 flowering plants and comparing the characteristics conserved and non-conserved lncRNAs in A. thaliana. The third part of the pipeline was to study the function of conserved and tissuespecific lncRNAs through the construction of a coexpression network.

\section{Data sources}

FASTA sequences for the lncRNAs from 25 plants were downloaded from CANTATAdb2.0 [17], GreeNC v1.12 [18], RefSeq [19], and NONCODEv5 [20] (Additional file 5). Genomic sequences and their corresponding annotations were retrieved from the NCBI genome database [19] (Additional file 1). An RNA-Seq dataset for 90 samples in nine tissues, including leaf, cotyledons, floral bud, seedling, seed, root, endosperm, inflorescence and silique was acquired from the NCBI SRA database [21] (Additional file 6). 


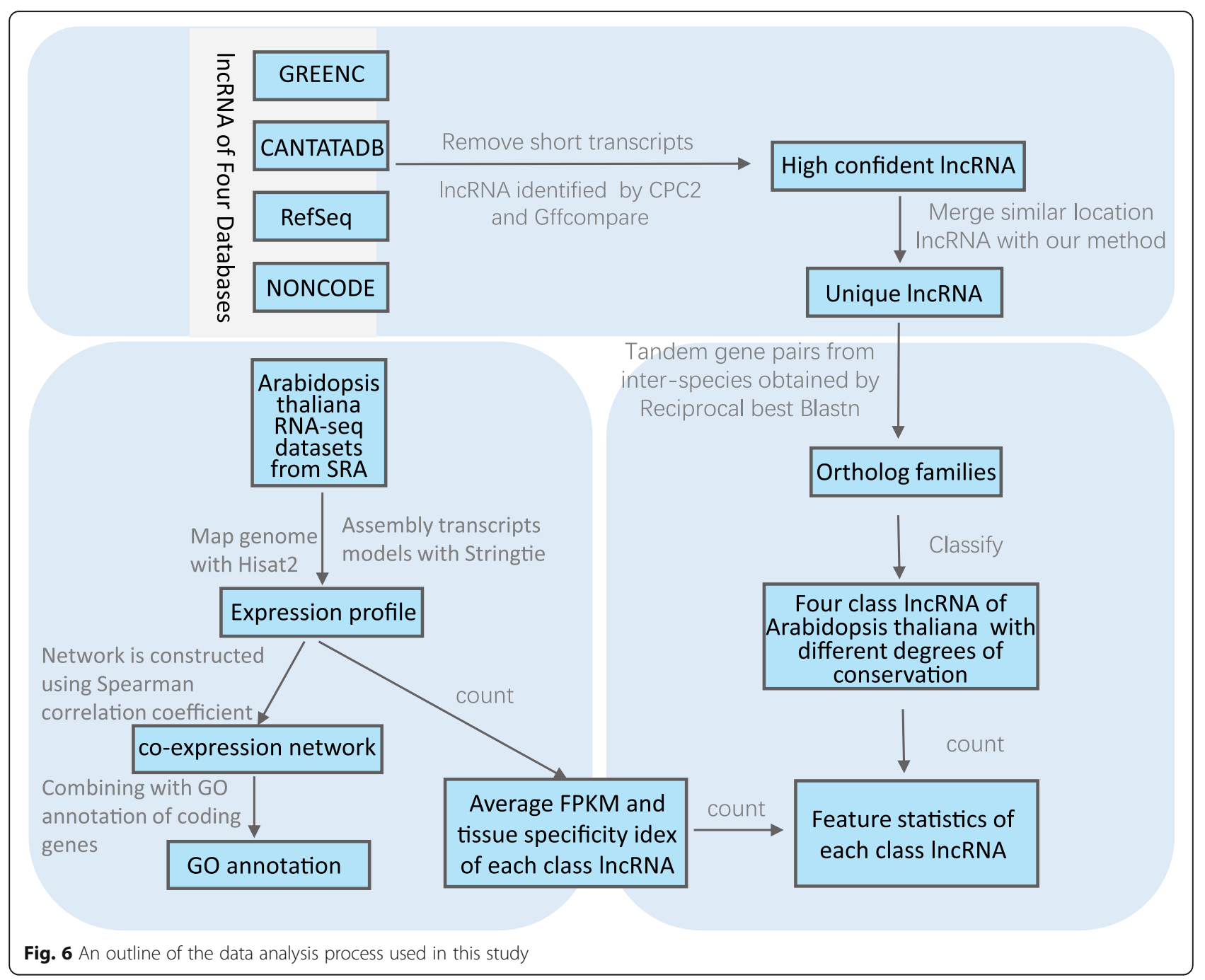

\section{Data integration}

All collected lncRNAs were filtered by CPC2 (coding potential) with default parameters and Gffcompare (v0.11.2) (genome location) with an optional parameters "-r" [22, 23]. Then, lncRNAs of each species were mapped to the selected genome version of that species (BLASTN, e-value $=10-5)$. The clustering method was used to determine the location of each lncRNA on the genome. The fragments of each lncRNA blast results, with a distance of no more than $4000 \mathrm{bp}$, were clustered, and the effective coverage and weighted identity of each cluster were calculated. We first selected clusters with the effective coverage greater than $70 \%$ and the weighted identity greater than $80 \%$, and the clusters with the highest effective coverage under the premise of the highest weighted identity were regarded as the optimal clusters for that particular lncRNA. Multiple optimal clusters were considered paralogous genes. We obtained lncRNA location information based on their optimal clusters so we could merge lncRNAs with overlapping lengths that were greater than $80 \%$ of the shorter lncRNA. If multiple lncRNAs were merged, one theoretical lncRNA, containing the union set of these lncRNA exons, was obtained.

\section{Identification of orthologous families and phylogenetic tree construction}

Orthologous gene-pairs were identified based on reciprocal best hits $(\mathrm{RBH})$ with BlastN using a relatively nonstringent E-value threshold of 10-5. The single-linkage clustering method was used to link gene-pairs together to establish orthologous families [7]. Each orthologous family was traced to the nearest branch point of the phylogenetic tree made by TimeTree, the nearest branch point meant that the species at this branch point were the smallest set which could contain all species in the orthologous family [13]. The final step was counting the number of orthologous families per branch point, and adding these numbers onto the phylogenetic tree. 


\section{RNA-seq data analysis pipeline}

The SRA-Toolkit was employed to convert SRA format files to FASTQ format files and low quality reads were trimmed using Trimmomatic (Version 0.39) [24]. RNASeq reads were aligned to the reference genome using Hisat2 (version 2.1.0) with the parameters of "--minintronlen 20" and "--max-intronlen 4000." Stringtie (v1.3.6) was used to calculate lncRNA- and coding-gene expression levels in each sample, which was measured by fragments per kilobase of transcript per million fragments sequenced (FPKM) and scaled by upper-quartile normalization $[14,25]$.

Using expression profiles of all SRA runs, the average expression level and the tissue specificity index of each lncRNA was calculated using average expression levels (AEL) (Eq. 1) and $\tau$ value (Eq. 2) [26].

$\mathrm{AEL}=\frac{\mathrm{AEL}_{\mathrm{l}}+\mathrm{AEL}_{\mathrm{c}}+\mathrm{AEL}_{\mathrm{f}}+\mathrm{AEL}_{\mathrm{sl}}+\mathrm{AEL}_{\mathrm{sd}}+\mathrm{AEL}_{\mathrm{r}}+\mathrm{AEL}_{\mathrm{e}}+\mathrm{AEL}_{\mathrm{i}}+\mathrm{AEL}_{\mathrm{sq}},}{9}$,

The nine parts of the numerator are the expression profile component values of each IncRNA expressed in samples of leaf, cotyledons, floral-bud, seedling, seed, root, endosperm, inflorescence and silique. Each component value was obtained by calculating the mean value of technical repeats and the median value of biological repeats. $A E L$ and $\mathrm{\tau}$ value were only calculated when at least one expression profile component of the expression profile had a value greater than or equal to 0.5 .

$$
\tau=\frac{\sum_{i=1}^{N}\left(1-x_{i}\right)}{N-1}
$$

where $N$ is the number of tissues and $x_{i}$ is the expression profile component normalized by the maximal component value. For example, expression profile ' $\begin{array}{lllllllllllll}8 & 0 & 0 & 0 & 2 & 0\end{array}$ $\begin{array}{lllll}2 & 0 & 0 & 0 & 0\end{array}$ ' is defined to have $\tau=0.95$. Other definitions, for example, based on entropy or geometric considerations, were pursued but found to be less robust in terms of sensitivity to extreme profile component values.

\section{Coding-IncRNA gene co-expression network construction}

The coding and non-coding genes with high expression variation (top $75 \%$ percentile) were retained to construct co-expression network. We employed an in-house Perl script to calculate the Spearman correlation coefficient and its corresponding $P$ value between the expression profiles of each gene-pair $[14,25]$. Only gene-pairs with an adjusted $P$ value of 0.05 or less were considered to be co-expressed.

\section{LncRNA functional annotation and enrichment}

GO annotation of $A$. thaliana coding gene was downloaded from the Gene Ontology Consortium (only biological process annotations were considered). GO annotation and enrichment of lncRNAs was predicted using goatools (version 0.9.5) [27], which determines the GO annotations of one gene based on the GO annotations of its immediate neighbor genes, and determines enriched GO annotations of study gene list based on the $\mathrm{GO}$ annotations of its population gene list $(P$-value < 0.05). Both GO annotation and enrichment are based on hypergeometric distribution (Eq. 3).

$$
P=1-\sum_{i=0}^{k-1} \frac{\left(\frac{M}{i}\right)\left(\frac{N-M}{n-\mathrm{i}}\right)}{\frac{N}{n}},
$$

For GO annotation, where $N$ is the total number of genes in the network, $M$ is the total number of genes with at least one GO annotation; $n$ is the number of a gene's immediate neighbors and $k$ is the number of neighbor genes with at least one $\mathrm{GO}$ annotation. For GO enrichment, where $N$ is the total number of annotated genes in the population, $M$ is the total number of genes annotated by one certain GO term; $n$ is the number of annotated genes in the study and $k$ is the number of genes annotated by one certain GO term.

\section{Abbreviations}

IncRNA: Long non-coding RNA; RBH: Reciprocal best hits; AEL: Average expression level; FPKM: Fragments per kilobase million; GO: Gene ontology; BP: Biological process

\section{Supplementary Information}

The online version contains supplementary material available at https://doi. org/10.1186/s12864-021-08047-6.

Additional file 1. Genomic sequences and their corresponding annotations of 25 flowering plants.

Additional file 2. Conserved IncRNAs ratios for each species.

Additional file 3. GO annotations of 196 conserved IncRNAs.

Additional file 4 Significant enrichment functions $(n=39)$ of conserved IncRNA set $(n=196)$.

Additional file 5. Download path of FASTA sequences for the IncRNAs from 25 plants.

Additional file $\mathbf{6}$. The collected transcriptome analysis data.

\section{Acknowledgments}

We thank all colleagues in our laboratory for helpful discussions and technical assistance.

\section{About this supplement}

This article has been published as part of BMC Genomics Volume 22 Supplement 3, 2021: 19th International Conference on Bioinformatics 2020 (InCoB2020): genomics. The full contents of the supplement are available online at https://bmcgenomics.biomedcentral.com/articles/supplements/ volume-22-supplement-3.

\section{Authors' contributions}

C.L. conceived and supervised this study. C.L. and S.S. designed the experiments. S.S., W.C. and D.Z. performed the experiments, analyzed and interpreted the data. X.Z. and W.Y. participated in the discussion and provided valuable advice and practical contributions. C.L. and S.S. wrote the manuscript. All authors reviewed, edited, and approved the final manuscript. 


\section{Funding}

This study was financially supported by the National Natural Science Foundation of China (No. 31970609), the Start-up Fund of Xishuangbanna Tropical Botanical Garden, and the 'Top Talents Program in Science and Technology' from Yunnan Province. Publication costs are funded by the National Natural Science Foundation of China (No. 31970609). The funders had no role in the design, data collection and analysis, decision to publish, or preparation of the manuscript.

\section{Availability of data and materials}

FASTA sequences for the IncRNAs are available from CANTATAdb2.0 (https:// cantata.amu.edu.pl, https://yeti.amu.edu.pl/CNATATA/), GreeNCv1.12 (https:// greenc.sciencedesigners.com/), RefSeq (https://ftp.ncbi.nlm.nih.gov/genomes/ refseq/plant/), and NONCODEv5 (http://www.noncode.org/). More detailed download paths for IncRNA sequences of each species can be found in Additional file 5. The processed IncRNA data and data processing scripts are available at https://github.com/changningliu-lab/sang-paper-2021. Genomic sequences and their corresponding annotations were retrieved from the NCBI genome database (https://ftp.ncbi.n/m.nih.gov/genomes/refseg/plant/, Additional file 1). The RNA-Seq dataset was acquired from the NCBI SRA database (https://trace.ncbi.nlm.nih.gov/Traces/sra/, Additional file 6).

\section{Declarations}

Ethics approval and consent to participate

Not applicable.

\section{Consent for publication}

Not applicable.

\section{Competing interests}

The authors declare that they have no competing interests.

\section{Author details}

${ }^{1}$ CAS Key Laboratory of Tropical Plant Resources and Sustainable Use, Xishuangbanna Tropical Botanical Garden, Chinese Academy of Sciences, Menglun, Mengla 666303, Yunnan, China. ${ }^{2}$ College of Life Sciences, University of Chinese Academy of Sciences, Beijing 100049, China. ${ }^{3}$ Center of Economic Botany, Core Botanical Gardens, Chinese Academy of Sciences, Menglun, Mengla 666303, Yunnan, China. ${ }^{4}$ The Innovative Academy of Seed Design, Chinese Academy of Sciences, Menglun, Mengla 666303, Yunnan, China.

Received: 28 September 2021 Accepted: 30 September 2021 Pulished: 14 October 2021

\section{References}

1. Ma L, Bajic VB, Zhang Z. On the classification of long non-coding RNAs. RNA Biol. 2013;10(6):925-33. https://doi.org/10.4161/rna.24604.

2. Li J, Zhang X, Liu C. The computational approaches of IncRNA identification based on coding potential: status quo and challenges. Comput Struct Biotechnol J. 2020;18:3666-77. https://doi.org/10.1016/j.csbj.2020.11.030,

3. Bonidia RP, Machida JS, Negri TC, Alves WAL, Kashiwabara AY, Domingues DS, et al. A novel decomposing model with evolutionary algorithms for feature selection in long non-coding RNAs. IEEE Access. 2020;8:181683-97. https://doi.org/10.1109/ACCESS.2020.3028039.

4. Gibb EA, Brown CJ, Lam WL. The functional role of long non-coding RNA in human carcinomas. Mol Cancer. 2011;10(1):38. https://doi.org/10.1186/14 76-4598-10-38

5. Wu L, Liu S, Qi H, Cai H, Xu M. Research progress on plant long non-coding RNA. Plants. 2020;9(4):408. https://doi.org/10.3390/plants9040408.

6. Washietl S, Kellis M, Garber M. Evolutionary dynamics and tissue specificity of human long non-coding RNAs in six mammals. Genome Res. 2014;24(4): 616-28. https://doi.org/10.1101/gr.165035.113

7. Necsulea A, Soumillon M, Warnefors M, Liechti A, Daish T, Zeller U, et al. The evolution of IncRNA repertoires and expression patterns in tetrapods. Nature. 2014;505(7485):635-40. https://doi.org/10.1038/nature12943.

8. Hezroni H, Koppstein D, Schwartz MG, Avrutin A, Bartel DP, Ulitsky I. Principles of long non-coding RNA evolution derived from direct comparison of transcriptomes in 17 species. Cell Rep. 2015;11(7):1110-22. https://doi.org/10.1016/j.celrep.2015.04.023.
9. Liu J, Jung $C, X u$ J, Wang H, Deng S, Bernad L, et al. Genome-wide analysis uncovers regulation of long intergenic non-coding RNAs in Arabidopsis. Plant Cell. 2012;24(11):4333-45. https://doi.org/10.1105/tpc.112.102855.

10. Li L, Eichten SR, Shimizu R, Petsch K, Yeh C, Wu W, et al. Genome-wide discovery and characterization of maize long non-coding RNAs. Genome Biol. 2014;15(2):R40. https://doi.org/10.1186/gb-2014-15-2-r40.

11. Deng P, Liu S, Nie X, Song W, Wu L. Conservation analysis of long noncoding RNAs in plants. Sci China Life Sci. 2018;61(2):190-8. https://doi.org/1 0.1007/s11427-017-9174-9.

12. Camacho C, Coulouris G, Avagyan V, Ma N, Papadopoulos J, Bealer K, et al. BLAST+: architecture and applications. BMC Bioinform. 2009;10(421):1-9. https://doi.org/10.1186/1471-2105-10-421.

13. Kumar $S$, Stecher $G$, Suleski M, Hedges S. TimeTree: a resource for timelines, timetrees, and divergence times. Mol Biol Evol. 2017;34(7):1812-9. https:// doi.org/10.1093/molbev/msx116.

14. Chen W, Zhang X, Li J, Huang S, Xiang S, Hu X, et al. Comprehensive analysis of coding-IncRNA gene co-expression network uncovers conserved functional IncRNAs in zebrafish. BMC Genomics. 2018;19(Suppl 2):112. https://doi.org/10.1186/s12864-018-4458-7.

15. Ulitsky I. Evolution to the rescue: using comparative genomics to understand long non-coding RNAs. Nat Rev Genet. 2016;17(10):601-14. https://doi.org/10.1038/nrg.2016.85.

16. Chen J, Shishkin A, Zhu X, Kadri S, Maza I, Guttman M, et al. Evolutionary analysis across mammals reveals distinct classes of long non-coding RNAs. Genome Biol. 2016;17(1):19. https://doi.org/10.1186/s13059-016-0880-9.

17. Szczesniak MW, Bryzghalov O, Ciomborowska-Basheer J, Makałowska I. CANTATAdb 2.0: expanding the collection of plant long non-coding RNAs. Methods Mol Biol. 2019;1933:415-29. https://doi.org/10.1007/978-1-4939-904 5-0_26.

18. Paytuvi Gallart A, Hermoso Pulido A, Anzar Martínez de Lagrán I, Sanseverino W, Aiese Cigliano R. GREENC: a Wiki-based database of plant IncRNAs. Nucleic Acids Res. 2016;44(D1):D1161-6. https://doi.org/10.1093/na r/gkv1215.

19. Pruitt KD, Tatusova T, Brown GR, Maglott DR. NCBI reference sequences (RefSeq): current status, new features and genome annotation policy. Nucleic Acids Res. 2012;40(D1):D130-5. https://doi.org/10.1093/nar/gkr1079.

20. Fang S, Zhang L, Guo I, Wu Y, Li H, Zhao L, et al. NONCODEV5: a comprehensive annotation database for long non-coding RNAs. Nucleic Acids Res. 2018;46(D1):D308-14. https://doi.org/10.1093/nar/gkx1107.

21. Kodama $Y$, Shumway $M$, Leinonen $R$, on behalf of the International Nucleotide Sequence Database Collaboration. The sequence read archive: explosive growth of sequencing data. Nucleic Acids Res. 2012;40(D1):D54-6. https://doi.org/10.1093/nar/gkr854.

22. Kang YJ, Yang DC, Kong $L$, Hou $M$, Meng YQ, Wei $L$, et al. CPC2: a fast and accurate coding potential calculator based on sequence intrinsic features. Nucleic Acids Res. 2017:45(W1):W12-6. https://doi.org/10.1093/nar/gkx428.

23. Pertea G, Pertea M. GFF utilities: GffRead and GffCompare. F1000Res. 2020;9: 304.

24. Bolger AM, Lohse M, Usadel B. Trimmomatic: a flexible trimmer for Illumina sequence data. Bioinform. 2014;30(15):2114-20. https://doi.org/10.1093/ bioinformatics/btu170.

25. Liao Q, Liu C, Yuan X, Kang S, Miao R, Xiao H, et al. Large-scale prediction of long non-coding RNA functions in a coding-non-coding gene coexpression network. Nucleic Acids Res. 2011;39(9):3864-78. https://doi.org/1 $0.1093 / \mathrm{nar} / \mathrm{gkq} 1348$.

26. Yanai I, Benjamin H, Shmoish M, Chalifa-Caspi V, Shklar M, Ophir R, et al. Genome-wide midrange transcription profiles reveal expression level relationships in human tissue specification. Bioinform. 2005;21(5):650-9. https://doi.org/10.1093/bioinformatics/bti042.

27. Klopfenstein DV, Zhang L, Pedersen BS, Ramirez F, Warwick Vesztrocy A, Naldi A, et al. GOATOOLS: a python library for gene ontology analyses. Sci Rep. 2018;8(1):10872. https://doi.org/10.1038/s41598-018-28948-z.

\section{Publisher's Note}

Springer Nature remains neutral with regard to jurisdictional claims in published maps and institutional affiliations. 